\title{
Mechanism of Yanghe Pingchuan granules treatment for airway remodeling in asthma
}

This article was published in the following Dove Press journal:

Drug Design, Development and Therapy

\author{
LingYu Pan ${ }^{1, *}$ \\ YanQuan Han 1,* \\ YongZhong Wang' \\ QianQian Chen ${ }^{2}$ \\ Ying $\mathrm{Wu}^{2}$ \\ Yuan Sun ${ }^{2}$
}

'Grade 3 Preparation Laboratory of State Administration of TCM, The First Affiliated Hospital of Anhui University of Chinese Medicine, Hefei, Anhui 23003I, China; ${ }^{2}$ Anhui University of Chinese Medicine, Hefei, Anhui 23003I, China

*These authors contributed equally to this work
Correspondence: YongZhong Wang The First Affiliated Hospital, Anhui University of Chinese Medicine, No. I17, Meishan Road, Hefei, Anhui 23003I,

China

Tel/fax +86 55। 62838553

Email wyzhmail@।63.com
Purpose: Yanghe Pingchuan granules (YPG), a hospital preparation developed by The First Affiliated Hospital, Anhui University of Chinese Medicine, has been used for the clinical treatment of bronchial asthma (BA) for several decades. This study aimed to explore the mechanism of action of YPG in the treatment of BA.

Materials and methods: Male Sprague Dawley rats $(n=60)$ were randomly divided into six groups ( $\mathrm{n}=10$ per group): control, a BA model, positive drug control (Guilong Kechuanning capsules; a proven effective treatment for BA), and model rats treated with a high, medium, or low dose of YPG. H\&E staining was used to detect pathological changes in the bronchial tubes. The mRNA expression levels of $P I 3 K, P K B, P C N A$, and $A R$ were determined by realtime PCR, and the protein levels of phospho- (p-)PI3K, p-PKB, p-PCNA, and p-AR were detected by Western blotting. ELISAs were used to detect the expression of PIP2, PIP3 IL-6, IL-8, IL-1 $\beta$, and epinephrine (EPI).

Results: H\&E staining demonstrated that BA can be ameliorated using YPG. Real-time PCR, Western blotting, and ELISA indicated that use of YPG decreased expression of the phosphoinositide 3-kinase (PI3K) signaling pathway and PCNA, and can also ameliorate the condition kidney Yang deficiency, which is associated with BA in Chinese traditional medicine.

Conclusion: YPG can attenuate BA therapeutically in a dose-dependent manner. The mechanism underlying its therapeutic effect comprises influences on three features that contribute to BA: the PI3K signaling pathway, cell proliferation, and "kidney-Yang deficiency".

Keywords: bronchial asthma, YPG, PI3K/PKB signaling pathway, cell proliferation, kidney Yang deficiency

\section{Introduction}

Bronchial asthma (BA) is a type of allergic disease that seriously compromises the health of affected individuals worldwide. BA occurs among young adults and children, and is hard to differentiate from wheezing as a result of viral infections. In addition, it is necessary to take ethnic differences into account. ${ }^{1,2}$ The incidence of asthma increases almost every year, and has doubled since $1980 .^{3}$

BA is a complex chronic disease characterized by variable airway limitation, airway remodeling, and airway hyper-reactivity. ${ }^{4}$ The pathogenesis of asthma has been considered to be caused by an imbalance of Th2-type and Th1-type lymphocytes. ${ }^{5}$ An increase of Th2 cells and release of cytokines are the two factors underlying airway injury. ${ }^{6}$ Currently, the primary pathogenesis of BA is considered to arise from an imbalance of Th-type lymphocytes, airway inflammation, airway remodeling, and the regulation of signaling pathways. Eventually, the airway inflammation leads to airway remodeling. Airway remodeling is a principal pathological feature of BA; therefore, it is necessary to study its pathogenesis. 
Airway remodeling is associated with the abnormal proliferation of airway smooth muscle cells (ASMCs). The manifestations of airway remodeling include glandular hypertrophy, goblet cells, hyperplasia of smooth muscle fibers, and basement membrane thickening. ${ }^{7}$ ASMCs can secrete various factors, including matrix proteins, growth factors, cytokines, and other molecules, to modulate airway inflammation and promote airway remodeling. ${ }^{8}$ The activation and proliferation of ASMCs is influenced by numerous factors, including cytokines, growth factors, extracellular matrix proteins, and signaling pathways. Phosphoinositide 3-kinase (PI3K) signaling has a clear role in the proliferation of ASMCs, significantly influencing the regulation of ASMC proliferation. ${ }^{9}$ The PI3K pathway has essential functions in various inflammatory cells, including control of cell growth and survival, along with modulation of cytoskeleton remodeling. Upon activation, PI3K converts phosphatidylinositol $(3,4)$ bisphosphate (PI $(3,4)$ P2) to phosphatidylinositol $(3,4,5)$ trisphosphate (PI $(3,4,5) \mathrm{P} 3)$, which activates downstream signaling molecules with regulatory roles in cells. PKB is a serine/threonine protein kinase downstream of the PI3K signaling pathway. ${ }^{10}$ Once activated, PKB can accelerate the cell cycle and promote DNA replication to stimulate the proliferation of ASMCs and exacerbate airway remodeling. The proliferation of ASMCs and airway remodeling are strongly correlated with PCNA, a nuclear protein indispensable for the synthesis of DNA, a process key to cell proliferation and cycling. ${ }^{11}$ As an accurate, direct, and straightforward indicator of cell proliferation, PCNA can be used to assess the severity of airway remodeling.

In traditional Chinese medicine (TCM), a classical and characteristic concept is that of the use of the "same treatments for different diseases". ${ }^{12}$ In TCM, asthma is considered to be caused, not only by the lungs, but also by the kidney; hence the condition kidney Yang deficiency is established as contributing to the disease. To translate the TCM concept of kidney Yang deficiency into modern medical concepts, it is necessary to clearly understand that the pathogenesis of asthma is strictly related to the function of the hypothalamicpituitary-adrenal (HPA) axis. ${ }^{13} \mathrm{~A}$ decrease in HPA axis function may aggravate airway inflammation in asthma; however, studies of the role of the kidney in the pathogenesis of asthma remain very rare. In this investigation we detected changes in epinephrine (EPI) and adrenergic receptor (AR) levels during asthma, thereby verifying the presence of kidney Yang deficiency during asthma.

Yanghe Pingchuan granules (YPG) is a hospital preparation made at The First Affiliated Hospital, Anhui University of Chinese Medicine. YPG contains nine types of TCM, as follows:

1) Ephedra sinica Stapf., an antipyretic and expectorant;

2) Rehmannia glutinosa (Gaertn.) DC, which helps to increase the blood volume and enhances renal function;

3) Inula japonica Thunb., which eases coughing and acts as an expectorant;

4) Morinda officinalis F.C.How, which is used to treat impotence and nocturnal emission;

5) Schisandra chinensis (Turcz.) Baill., which strengthens the body by tonifying the heart and kidney;

6) Sinapis alba L., which warms the lungs, dispels phlegm, and can relieve "Qi" and pain;

7) Draba nemorosa L., an expectorant, which also promotes the discharge of urine to eliminate edema;

8) Angelica sinensis (Oliv.) Diels, used to regulate menstruation, relieve pain, and prevent cancer; and

9) Platycodon grandiflorus (Jacq.) A.DC., which helps to relieve coughs and expel phlegm.

YPG can improve kidney Yang deficiency and cure BA. ${ }^{14,15}$ Over the last few decades, YPG has been used for effective clinical treatment of $\mathrm{BA} ; ;^{16,17}$ however, the mechanism underlying its action is not precisely understood. This paper evaluated the effectiveness, practicality, and feasibility of YPG for treatment of asthma and its effects on three physiological processes: PI3K signaling, cell proliferation, and kidney Yang deficiency.

\section{Materials and methods}

\section{Ethics statement}

We purchased male Sprague Dawley (SD) rats from the Laboratory Animal Center, Medical University of Anhui Province. All experiments were subject to approval by the Committee on the Ethics of Animal Experiments of Anhui University of Chinese Medicine (Permit Number: 2014AH029-02). All experiments were in accordance with the following rules and regulations:

1) The "Regulations for the Administration of Affairs Concerning Experimental Animals" issued by the National Science and Technology Commission.

2) The "Implementation Rules for the Management of Medical Laboratory Animals" issued by the Ministry of Health of the People's Republic of China.

All surgeries were performed under sodium pentobarbital anesthesia, and all efforts were made to minimize suffering.

\section{Chemicals and instruments}

Egg albumin (Sigma Aldrich Co., St Louis, MO, USA, batch number: A5378), Guilong Kechuanning capsules (Guilong Pharmaceutical (Anhui) Co. Ltd., Ma'Anshan, 
China, batch number: 150509); Yanghe Pingchuan granules (The First Affiliated Hospital, Anhui University of Chinese Medicine, batch number: 20161209).

\section{YPG preparation}

Herbal materials included E. sinica, R. glutinosa, I. japonica, M. officinalis, S. chinensis, S. alba, D. nemorosa, A. sinensis, and $P$. grandiflorus. After determination of the optimum extraction process, herbal materials were pulverized to a powder, vacuum dried at $70^{\circ} \mathrm{C}$, then eight volumes of $80 \%$ ethanol were added twice for $1.5 \mathrm{~h}$ each to extract active components. The extract was then filtered, and filtrates concentrated under reduced pressure until there was no alcohol odor.

\section{Analysis of YPG by UPLC-PDA}

Analysis of YPG quality was carried out using a Waters Acquity ultra-performance liquid chromatography (UPLC) H-Class system, consisting of an autosampler, a quaternary pump, and an Acquity photo-diode array (PDA) detector. An Acquity UPLC hybrids of ethyl bridge $(\mathrm{BEH}) \mathrm{C}_{18}$ column $(100 \times 2.1 \mathrm{~mm}, 1.7 \mu \mathrm{m})$ was used for all separations: column temperature $=35^{\circ} \mathrm{C}$; analysis time $=22 \mathrm{~min}$; flow rate $=0.3 \mathrm{~mL} / \mathrm{min}$; injection volume $=0.5 \mu \mathrm{L}$; and wavelength $=254 \mathrm{~nm}$. The mobile phase consisted of acetonitrile (A) and phosphoric acid (B). The gradient elution program was: $0-3 \min (5 \%-18 \% \mathrm{~A})$, $3-6 \min (18 \%-21 \% \mathrm{~A}), 6-10 \mathrm{~min}(21 \%-25 \% \mathrm{~A}), 10-10.5 \mathrm{~min}$ (25\%-50\% A), $10.5-13 \min (50 \%-75 \%$ A), $13-17 \mathrm{~min}$ (75\%-80\% A), 17-18 $\min (80 \%-90 \%$ A), $18-19 \min$ (100\%-5\% A), and 19-22 $\min (5 \%$ A). Five reference substances were used for qualitative analysis: sinapine thiocyanate, ferulic acid, acteoside, auercetin, and schisandrin. All reference substances were acquired from the National Institute for the Control of Drug and Biological Products (Beijing, China). Similarity Evaluation Software of Traditional Chinese Medicine Injection (National Pharmacopoeia Commission of China, Edition A, 2004) was used to analyze the results of UPLC chromatography.

\section{Animal experiments, establishment of a BA model, and sample collection}

After acclimatization for 1 week, SD rats were randomly divided into six groups ( $\mathrm{n}=10$ per group), as follows: healthy control, BA model, BA model treated with high (YPG H), medium (YPG M), and low (YPG L) dose YPG, and positive drug (Guilong Kechuanning capsule) control. On the first day, rats in the model, YPG H, YPG M, YPG L, and positive drug control groups were injected intraperitoneally with $10 \%$ egg albumin dissolved in $0.9 \%$ saline. After 2 weeks, all six groups (including the healthy control group) were aerosolized with $1 \%$ egg albumin dissolved in $0.9 \%$ saline five-times every 2 days, once a week, to induce BA. Hydrocortisone $(1 \mathrm{mg} / \mathrm{mL})$ was injected intraperitoneally on the 1st, 16th, 18th, 19th, 22nd, 26th, and 28th day, with doses of $1.0,1.2,1.4,1.6,2.0,8.0$, and $8.0 \mathrm{~mL}$, respectively, to produce the kidney deficiency model.

On the 28th day after modeling, rats in the YPG H, YPG $\mathrm{M}$, and YPG L groups were administered with YPG (14.76, 7.38 , and $3.69 \mathrm{~g} / \mathrm{kg}$, respectively, once a day) by gavage. The positive control group received Guilong Kechuanning capsules by gavage. Control and model animals were administered with an equivalent amount of regular saline once a day for 2 weeks. After the final dose, animals were anesthetized by intraperitoneal injection of $2 \%$ pentobarbital sodium (40 mg/kg), and bronchial tissue collected in freezing tubes and stored at $-80^{\circ} \mathrm{C}$.

\section{H\&E staining}

Right lungs were fixed, paraffin embedded, and sectioned. Changes in airway wall structure and pathological alterations of the bronchi and smooth muscles were observed by conventional H\&E staining and analyzed using ImageJ software.

\section{PI3K, PKB, PCNA, and AR mRNA expression}

Trizol reagent was used to extract RNA from bronchial tissue samples. cDNA was synthesized by reverse transcription using $2 \mu \mathrm{g}$ of total RNA as a template. $\beta$-actin was selected as an internal control. The relative expression levels of $P I 3 K$, $P K B, P C N A$, and $A R$ mRNA were determined by quantitative fluorescence PCR (ABI7000 quantitative fluorescence monitor) at $50^{\circ} \mathrm{C}$ for $2 \mathrm{~min}, 95^{\circ} \mathrm{C}$ for $15 \mathrm{~min}, 95^{\circ} \mathrm{C}$ for $15 \mathrm{~s}$, and $60^{\circ} \mathrm{C}$ for $1 \mathrm{~min}$. The primers used are listed in Table 1.

\section{$\mathrm{PI} 3 \mathrm{~K}, \mathrm{PKB}, \mathrm{PCNA}$, and $\mathrm{AR}$ protein expression}

Bronchial tissue samples were lysed in $1 \mathrm{~mL}$ of cell extraction buffer. Lysates were incubated in radio-immunoprecipitation assay buffer and kept at $4^{\circ} \mathrm{C}$, for $2 \mathrm{~h}$, followed by centrifugation at $12,000 \times \mathrm{g}$ for $30 \mathrm{~min}$ at $4^{\circ} \mathrm{C}$, and the supernatants collected. Total proteins were separated by sodium dodecyl sulfate polyacrylamide gel electrophoresis on $8 \%$ gels, transferred onto polyvinylidene fluoride membranes, and blocked in TBST (plus 5\% skim milk powder, pH 7.4, tris-buffered saline [TBS] with $0.24 \%$ Tween-20). Membranes were then incubated overnight at $4{ }^{\circ} \mathrm{C}$ with the following primary antibodies: p-PI3K (Abcam, ab86714, 1:1,000), p-PKB (Abcam, ab81283, 
Table I Primer sequences

\begin{tabular}{llll}
\hline $\begin{array}{l}\text { Gene } \\
\text { target }\end{array}$ & Sense primer (5' to $\left.\mathbf{3}^{\prime}\right)$ & Anti-sense primer (5' to $\mathbf{3}^{\prime}$ ) & $\begin{array}{l}\text { Product } \\
\text { length (bp) }\end{array}$ \\
\hline PI3K & ATACTTGATGTGGCTGACGC & AATCCTCGTCATCGTCTACC & 110 \\
$P K B$ & ACTCATTCCAGACCCACGAC & AGCCCGAAGTCCGTTATCTT & $24 I$ \\
$P C N A$ & ATGAGGCCTGCTGGGACATC & CGCAGCGGTATGTGTCGAAG & 117 \\
$A R$ & TTCTTCCTGGCCAACGTGGT & TTGAAGGCCGAGTTGGCGTA & 101 \\
$\beta$-actin & CAGCGGAACCGCTCATTGATGG & TCACCCACACTGTGCCCAACGA & 300 \\
\hline
\end{tabular}

1:5,000), p-PCNA (Abcam, ab92552, 1:3,000), p-AR (Abcam, ab3442, 1:1,000), and $\beta$-actin (Abcam, ab8226, 1:2,000). Blots were washed three-times for $15 \mathrm{~min}$ in TBST and treated with an horseradish peroxidase-conjugated polyclonal goat antirabbit antibody (Beyotime, A0208, 1:1,000) for $2 \mathrm{~h}$ at room temperature. Membranes were washed three-times for $10 \mathrm{~min}$, and HRP substrate used to detect the HRP-secondary antibody bound to the proteins of interest, based on the manufacturer's instructions. The $\mathrm{p}-\mathrm{PI} 3 \mathrm{~K}$, $\mathrm{p}-\mathrm{PKB}$, $\mathrm{p}-\mathrm{PCNA}$, and $\mathrm{p}-\mathrm{AR}$ protein expression levels were standardized to those of $\beta$-actin.

\section{PIP2, PIP3, IL-6, IL-8, IL-I $\beta$, and EPI expression}

Bronohoalveolarlavage fluid was collected from rats and centrifuged at $350 \mathrm{~g}$ for $5 \mathrm{~min}$ at $4^{\circ} \mathrm{C}$. Supernatants were stored at $-80^{\circ} \mathrm{C}$ for cytokine analysis. ELISAs were used to quantify the concentrations of PIP2 and PIP3, according to the manufacturer's instructions. OD values were read at 450 $\mathrm{nm}$, and the levels of PIP2, PIP3, IL-6, IL-8, IL-1 $\beta$, and EPI were determined based on standard curves.

\section{Statistical analysis}

Data are expressed as the mean \pm SD. Statistical analyses were performed using SPSS17.0 (SPSS, Chicago, IL, USA). One-way analysis of variance was performed for multiplesample analyses. $P<0.05$ was considered to be statistically significant.

\section{Results}

\section{Qualitative analysis of YPG by UPLC-PDA}

YPG was first analyzed by UPLC-PDA, alongside mixed reference standard compounds. As shown in Figure 1, over 16 peaks were generated in $23 \mathrm{~min}$. Comparisons of individual peak retention times with those of reference standard compounds identified five compounds, including sinapine thiocyanate (3), ferulic acid (5), acteoside (6), auercetin (7), and schisandrin (11) in YPG; other peaks were unknown.

\section{YPG treatment ameliorated $B A$ in vivo}

To explore the effect of YPG in BA, a model of BA was established, as described above. The results of histopathological

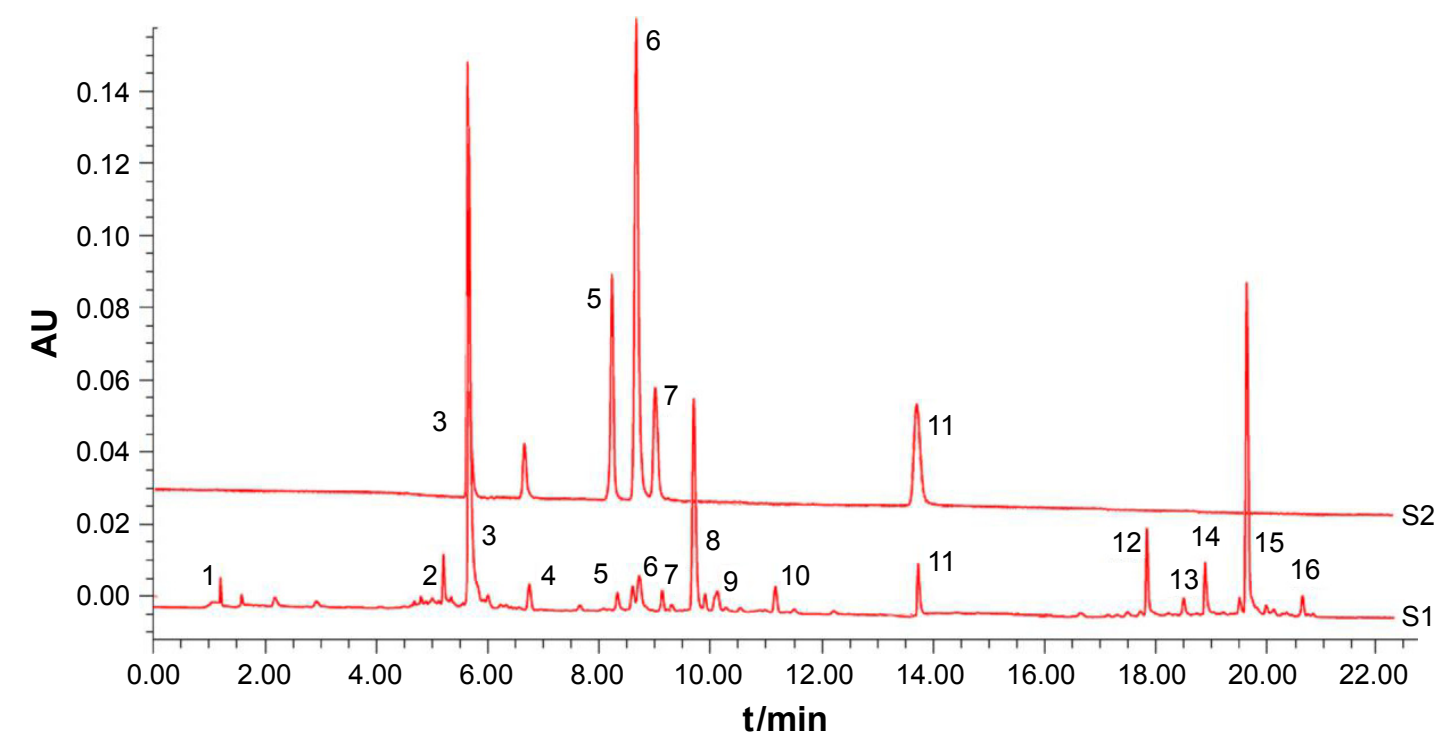

Figure I UPLC-PDA analysis of YPG.

Notes: Chromatograms of mixed reference standard compounds (SI) and YPG (S2). Sinapine thiocyanate (3), ferulic acid (5), acteoside (6), auercetin (7), and schisandrin (II) were identified by comparison with standards, while the other peaks represent unknown substances.

Abbreviations: UPLC, ultra-performance liquid chromatography; PDA, photo-diode array; YPG, Yanghe Pingchuan granules. 

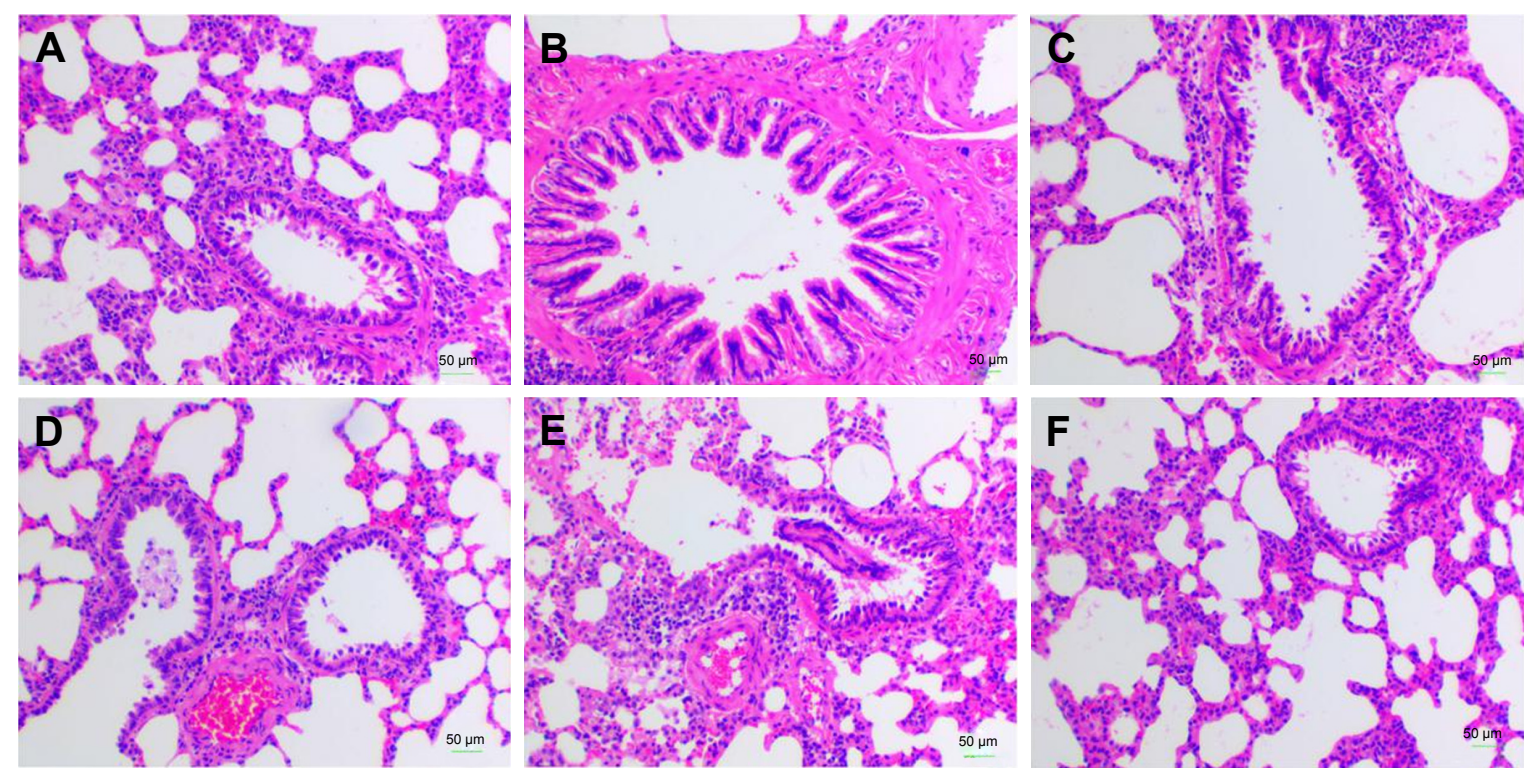

Figure 2 Effect of YPG on bronchial asthma in rats. Sections were stained with H\&E. Magnification, $\times 400$; scale bars, $50 \mu \mathrm{m}$. Healthy control group (A), Model group (B), YPG H group (C), YPG M group (D), YPG L group (E), positive control group (F).

Abbreviation: YPG, Yanghe Pingchuan granules.

evaluation of lung tissue samples from each of the six experimental groups (healthy control, BA model, YPG H, YPG M, YPG L, and positive control) are illustrated in Figure 2. In the healthy control group, there was no visible infiltration of inflammatory cells in the bronchus, the bronchial wall was smooth, the cells were arranged regularly, and the thickness of the soft muscle layer was even (Figure 2A). In contrast, a large number of eosinophils, monocytes, and lymphocytes infiltrated the bronchial tube wall in the model group. As shown in Figure 2B, a series of pathological changes were visible, including microvascular leakage, and goblet cell and airway epithelial cell hyperplasia. In addition, the smooth muscle layer thickened and the mucous membrane of the bronchial mucosa increased and extended. After treatment with YPG, infiltration of eosinophils, lymphocytes, and other inflammatory cells was significantly reduced; the thickness of the tube wall and the smooth muscle layer was close to that of the healthy control group; and the structures of the bronchial mucosa and trachea were improved (Figure 2C). The treatment effects in the YPG M and YPG L groups were less marked, because of the lower doses received by these animals (Figure 2D and E). In the positive control drug group, the submucosa showed mild congestion and contained few inflammatory cells, while inflammatory exudates were observed in the lumen, and the mucous membrane was increased (Figure 2F).

Compared with the healthy control group, the area to circumference ratio of the endobronchial tube and the rate of thickness to the boundary of smooth muscle in the model group were significantly increased $(P<0.01)$. These results suggested inflammation of the bronchi and soft tissues (Table 2). After treatment with YPG, the ratio decreased, and the therapeutic effect was dose-dependent $(P<0.01)$, with the effect of treatment in the YPG $\mathrm{H}$ group superior to those in the YPG M and L groups. Moreover, the effect of treatment in the YPG H group was similar to that observed in the positive control group.

\section{TIL-6/IL-8/IL-I $\beta$ expression}

To confirm the effect of treatment with YPG on BA, levels of IL-6, IL-8, and IL-1 $\beta$ were measured by ELISA (Figure 3). Compared with the healthy control group, the levels of IL-6, IL-8, and IL-1 $\beta$ were increased in the model group $(P<0.01)$.

Table 2 Airway pathological features in a rat bronchial asthma model and effect of YPG treatment

\begin{tabular}{lll}
\hline Group & $\begin{array}{l}\text { Area to circumference } \\
\text { ratio of the } \\
\text { endobronchial tube } \\
\left(\mu \mathrm{m}^{2} / \mu \mathrm{m}\right)\end{array}$ & $\begin{array}{l}\text { Rate of thickness } \\
\text { to the boundary } \\
\text { of smooth muscle } \\
\left(\mu \mathrm{m}^{2} / \mu \mathrm{m}\right)\end{array}$ \\
\hline Healthy control & $5.24 \pm 0.35$ & $2.46 \pm 0.20$ \\
Model & $13.64 \pm 0.92^{* *}$ & $8.52 \pm 0.49 * *$ \\
YPG H & $6.84 \pm 1.20^{\#}$ & $4.24 \pm 0.89^{\#}$ \\
YPG M & $7.63 \pm 0.40^{\#}$ & $4.71 \pm 0.23^{\#}$ \\
YPG L & $8.88 \pm 0.34^{\#}$ & $6.34 \pm 0.13^{\#}$ \\
Positive control & $7.14 \pm 0.42^{\#}$ & $4.76 \pm 0.30^{\#}$ \\
\hline
\end{tabular}

Notes: Data are presented as the mean $\pm S D$. $n=8$ per group. $* * p<0.0$ l, vs healthy control group; $\# P<0.01$, vs model; group $\mathrm{H}=$ high-dose, $\mathrm{M}=$ middle-dose, $\mathrm{L}=$ low-dose. Abbreviation: YPG, Yanghe Pingchuan granules. 

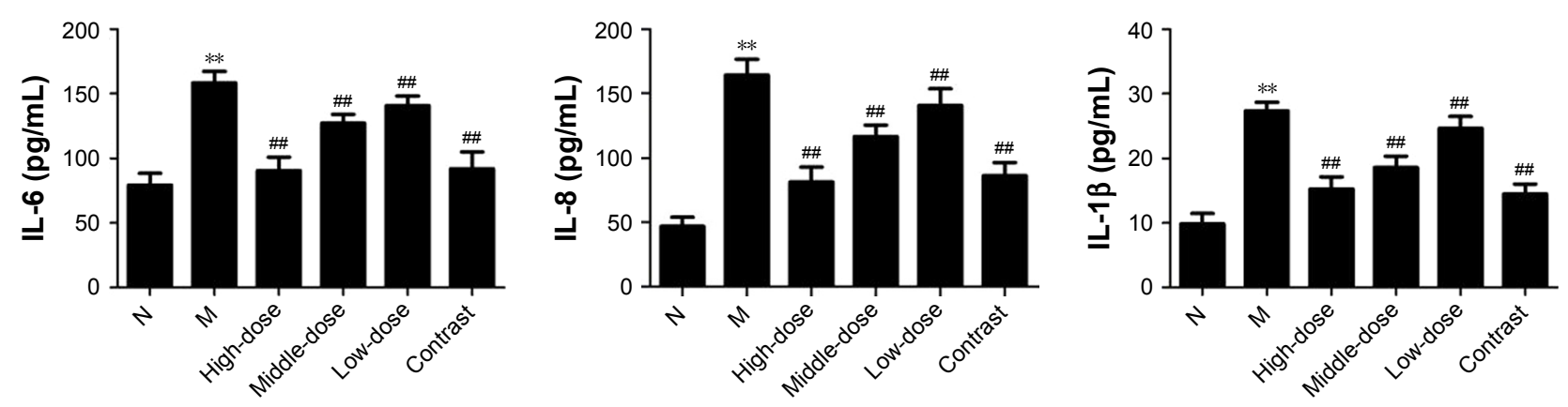

Figure 3 Effect of YPG on the expression of IL-6, IL-8, and IL-I $\beta$ in BA rats.

Notes: Levels of IL-6, IL-8, and IL-I $\beta$ were measured by ELISA. Data are expressed as means \pm SD. $n=8$. $* * P<0.0$ I vs healthy control $(\mathrm{N})$. ${ }^{*} P<0.0$ I vs model group $(\mathrm{M})$. Abbreviations: YPG, Yanghe Pingchuan granules; BA, bronchial asthma.

In groups treated with YPG, levels of IL-6, IL-8, and IL-1 $\beta$ were substantially reduced, and the therapeutic effect was dose-dependent $(P<0.01)$, with a superior effect observed in the YPG H group compared with the YPG M and L groups. The efficacy achieved in the YPG H group was similar to that observed in the positive control group. Together with the H\&E staining results, these results indicate that YPG can markedly improve features of BA in this model.

\section{YPG regulates ASMC proliferation} through targeting the PI3K/PKB pathway

To determine whether YPG treatment effects PI3K/PKB signaling in the context of BA, we evaluated the protein expression of $\mathrm{PI} 3 \mathrm{~K}$ and $\mathrm{PKB}$ in bronchial tissue from rats in all experimental groups. As shown in Figures 4 and 5, compared with the control group, the protein levels of PI3K and PKB were significantly increased after treatment with
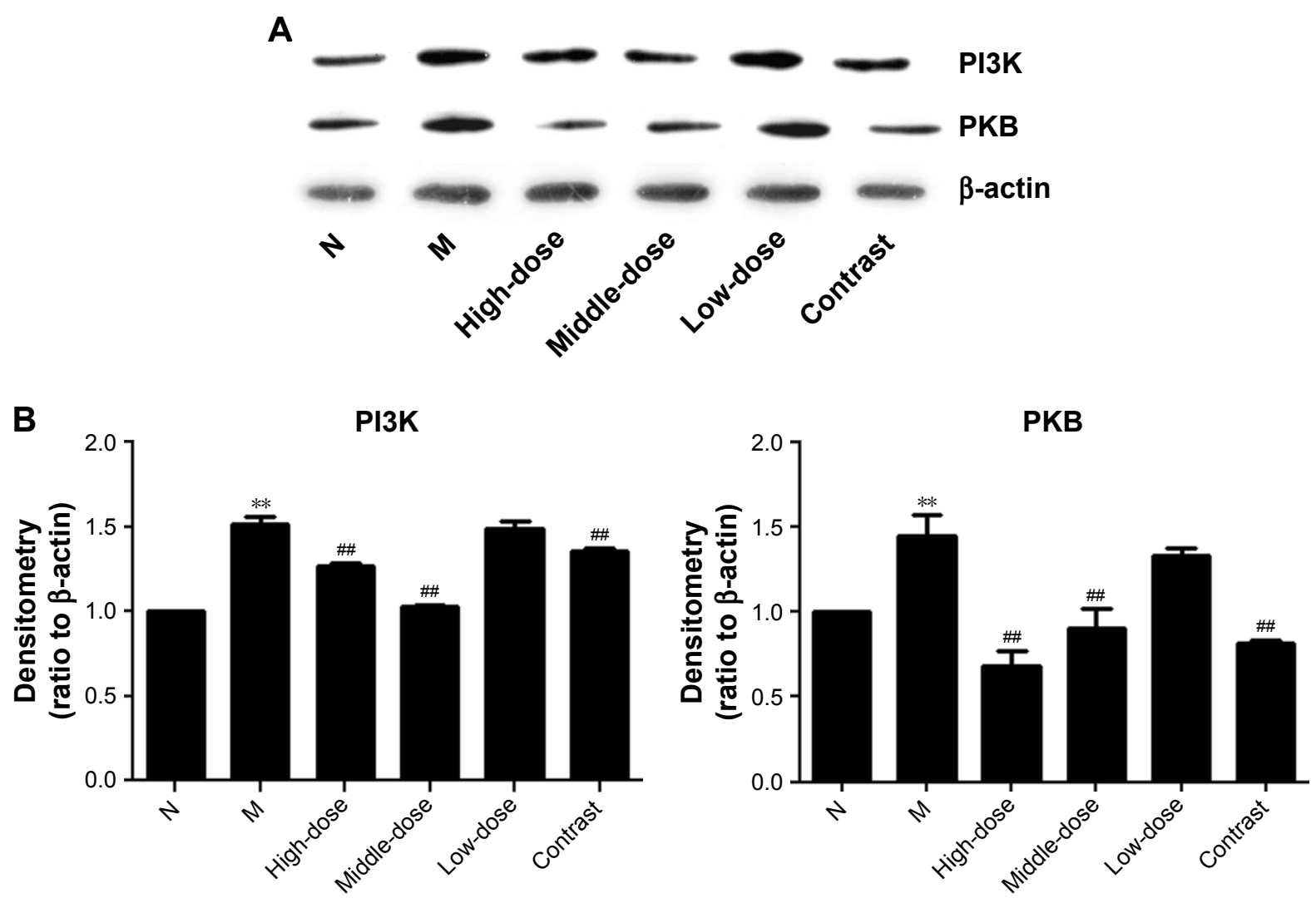

Figure 4 Effect of YPG on the expression of PI3K and PKB protein in BA rats. (A) Western blots demonstrating the expression levels of PI3K and PKB proteins. $\beta$-actin was used as a loading control. (B) Results of densitometry of Western blot bands.

Notes: Data are normalized to $\beta$-actin and expressed as means \pm SD. $n=3$. ${ }^{* * P} P<0.01$ vs healthy control (N); $P<0.05$ vs model group (M).

Abbreviations: YPG, Yanghe Pingchuan granules; BA, bronchial asthma. 

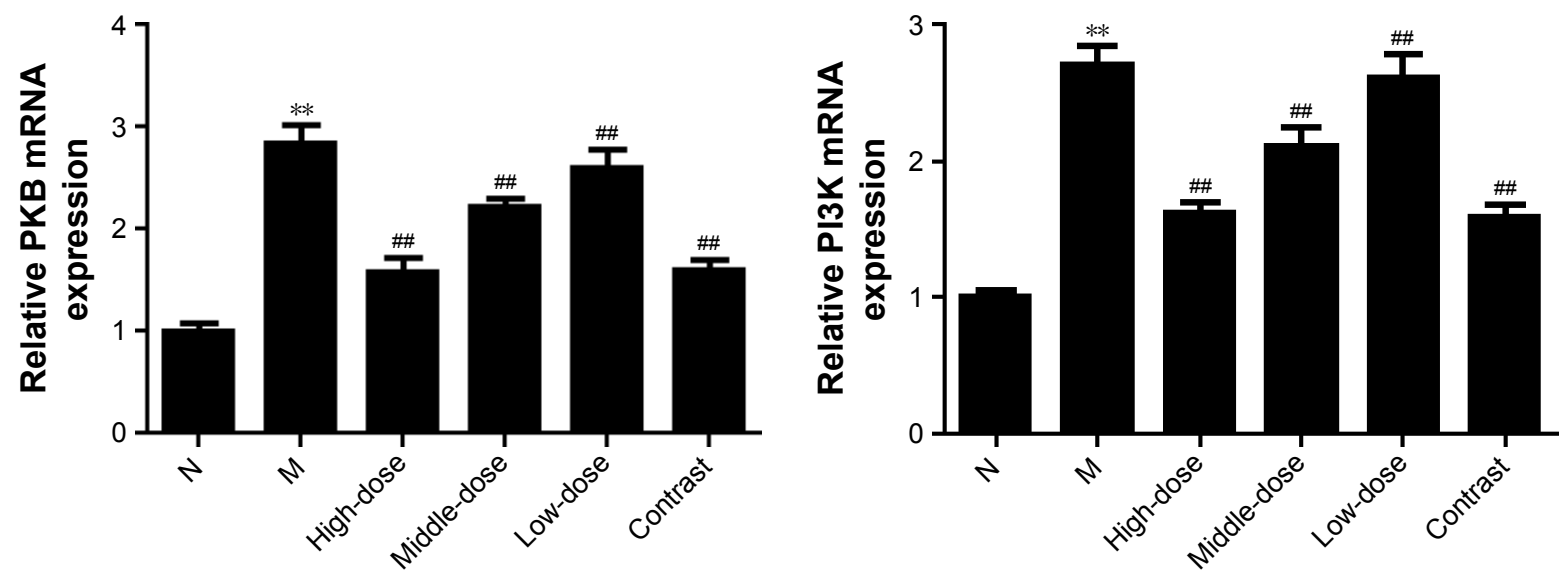

Figure 5 Effect of YPG on the expression of PI3K and PKB mRNA in BA rats.

Notes: mRNA levels of $P I 3 K$ and $P K B$ were determined by real-time $P C R$. Data are expressed as the mean $\pm S D$. $n=8$. $* * P<0.0$ I vs healthy control group (N); $P<0.01$ vs model group (M).

Abbreviations: YPG, Yanghe Pingchuan granules; BA, bronchial asthma.

YPG $(P<0.01)$, as were their mRNA levels. The inhibition effect on the PI3K pathway was not significant in the YPG L group; however, there were clear effects in the YPG $\mathrm{H}$ and L groups $(P<0.01)$.

\section{Effect of YPG on PIP2 and PIP3 expression} To further examine the effects of YPG on the PI3K/PKB signal pathway during treatment of BA, levels of the cytokines, PIP2 and PIP3, were monitored by ELISA. Compared with the healthy control group, levels of PIP2 and PIP3 were significantly increased in the model group $(P<0.01$; Figure 6). After YPG treatment, levels of PIP2 and PIP3 in the YPG-treated groups were decreased compared with the model group $(P<0.01)$. The most marked therapeutic effect was observed in the YPG H group, which had similar levels of cytokines to those in the positive control group.

\section{Evaluation of EPI and AR expression}

To test whether the onset of asthma is associated with kidney Yang deficiency, levels of EPI and AR were detected by ELISA, real-time PCR, and Western blot. As shown in Figure 7, compared with the healthy control group, levels of EPI and AR were decreased in the model group $(P<0.01)$. These data suggest that kidney function is decreased during asthma. After treatment with YPG, the expression levels of EPI and AR were significantly increased $(P<0.01)$, and $E P I$ and $A R$ mRNA levels in rats from the YPG $\mathrm{H}$ and positive control groups were restored to levels similar to those in the
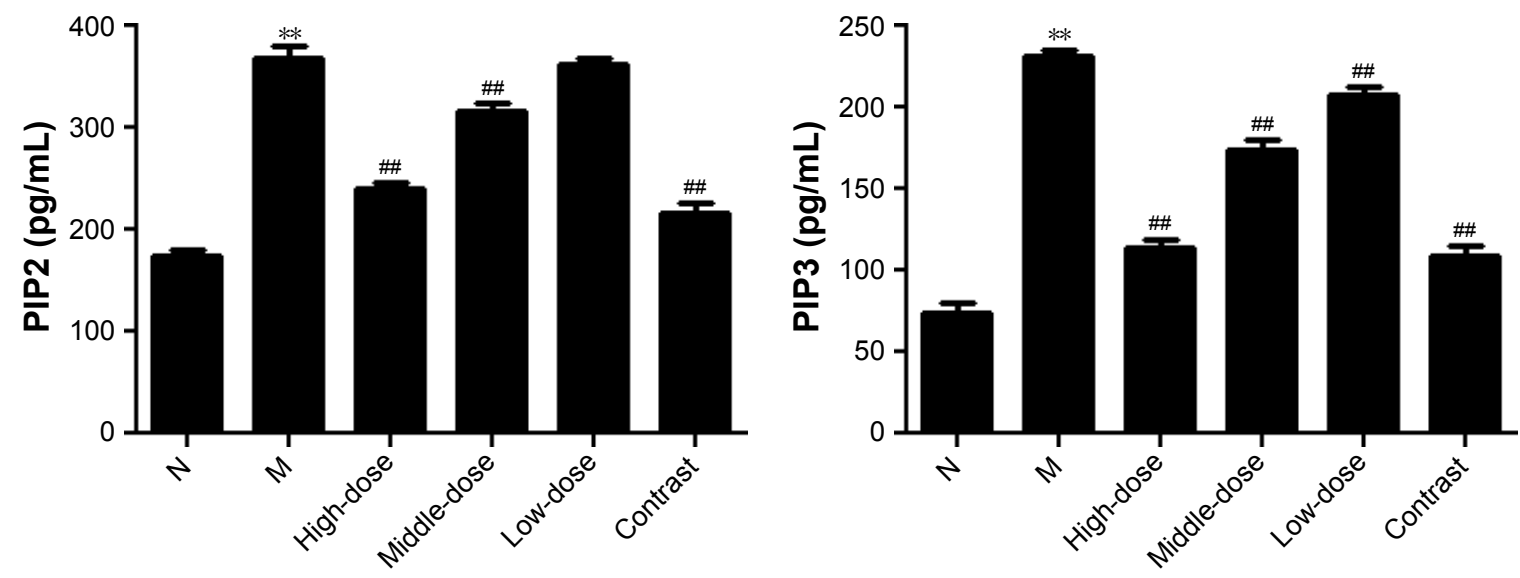

Figure 6 Effect of YPG on the expression of PIP2 and PIP3 in BA rats.

Notes: Levels of PIP2 and PIP3 were measured by ELISA. Data are expressed as means \pm SD. $n=8$. ${ }^{*} * P<0.01$ vs healthy control (N); ${ }^{\# P<0.0 I}$ vs model group (M). Abbreviations: YPG, Yanghe Pingchuan granules; BA, bronchial asthma. 
A

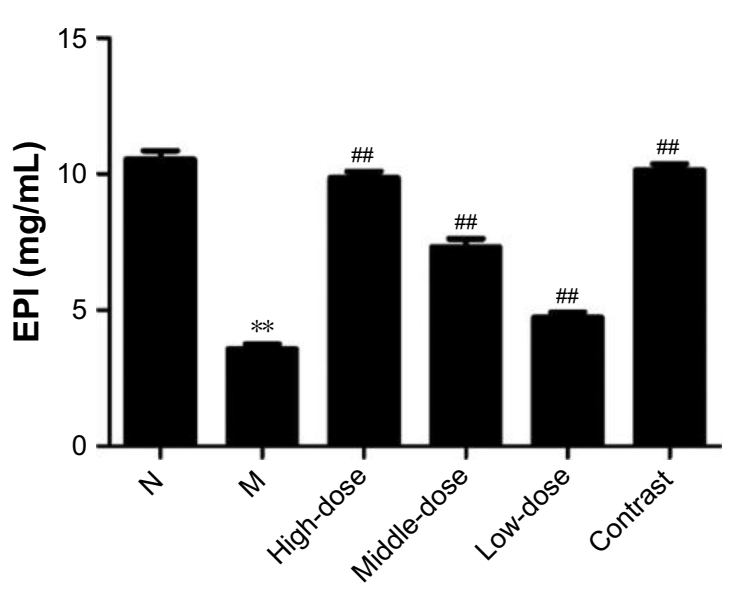

C

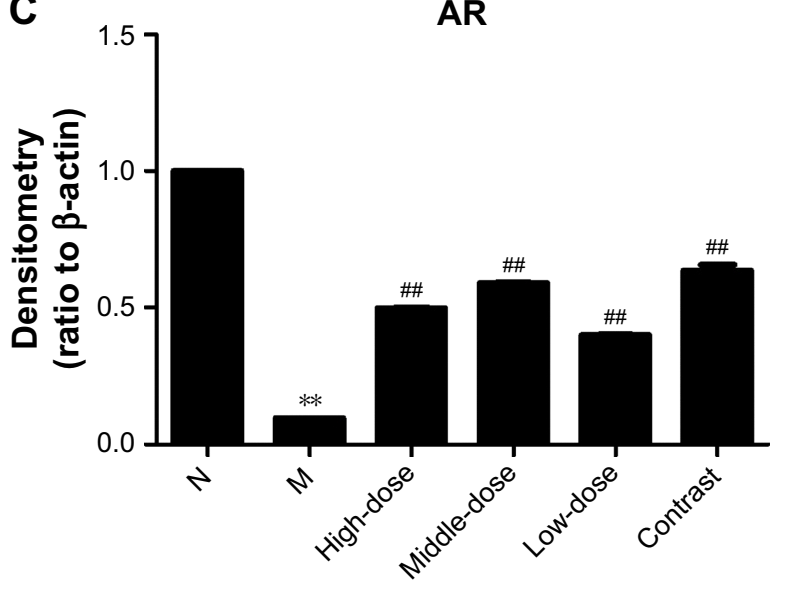

B

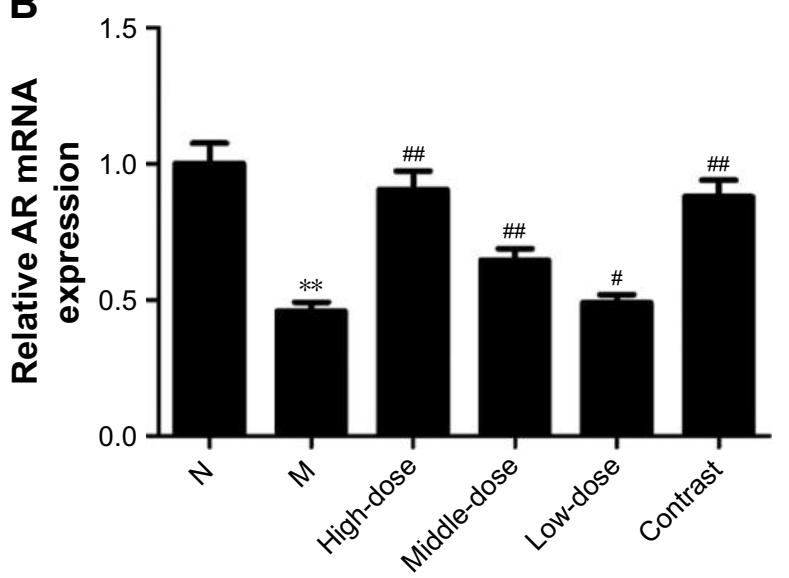

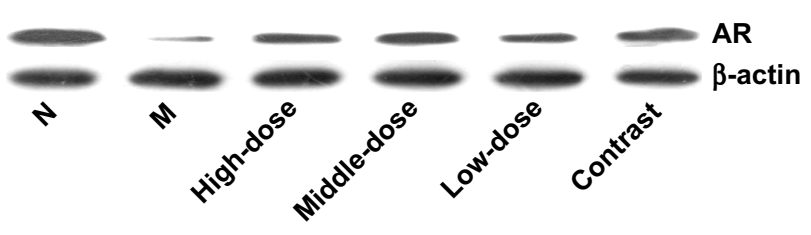

Figure 7 Effect of YPG on the expression of EPI and AR in BA rats. (A) Expression of EPI measured by ELISA; (B) Levels of AR mRNA were determined by real-time PCR. (C) Western blots to evaluate the expression of AR protein.

Notes: $\beta$-actin was used as a loading control. Densitometry band data were normalized to those of $\beta$-actin and are expressed as means \pm SD. $n=3$. $* * P<0.0 \mathrm{I}$ vs healthy

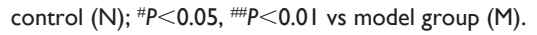

Abbreviations: YPG, Yanghe Pingchuan granules; EPI, epinephrine; AR, adrenergic receptor; BA, bronchial asthma.

healthy control group; however, AR protein levels were not restored by YPG treatment. These results indicate that YPG can improve kidney Yang deficiency.

\section{Evaluation of PCNA expression}

To test whether asthma is associated with abnormal cell proliferation, the expression of PCNA was detected by real-time PCR and Western blot. As shown in Figure 8, the expression of PCNA in the model group was significantly increased compared with the healthy control group $(P<0.01)$, indicating that asthma is accompanied by abnormal cell proliferation. After treatment with YPG, levels of PCNA decreased significantly $(P<0.01)$. A superior therapeutic effect was observed in the YPG H group compared with the YPG M and L groups, and treatment effects were dose-dependent.

\section{Discussion}

Asthma is an airway conduction disorder that responds to exogenous and endogenous stimuli in both children and adults. ${ }^{6}$ It is the most prevalent chronic respiratory disease, and resembles a complex syndrome, rather than a distinct disease. ${ }^{18}$ Asthma affects $\sim 300$ million peoples of all ages and ethnic groups, and from all countries, and is becoming a global health problem, leading to the deaths of 250,000 people annually. ${ }^{19}$ Asthma pathogenesis is complex, and involves a network of interacting factors at all levels and scales, from genetic to cellular, tissue, and organ. ${ }^{20}$ Airway remodeling is a primary cause of $\mathrm{BA}$ and a representative model of the condition. ${ }^{21}$ An increase in the number of ASMCs is a dominant structural change in asthma, and a crucial factor underlying airway remodeling and decreased lung function. ${ }^{22}$

The proliferation of ASMCs, which is a critical feature of airway remodeling, is correlated with the PI3K signaling pathway. ${ }^{23}$ In non-asthmatic ASMCs, mitogens control growth via the phosphoinositide 3-kinase (PI3K) and extracellular signal-regulated kinase (ERK) signaling pathways. ${ }^{24}$ The PI3K family of proteins controls a wide variety of cellular 

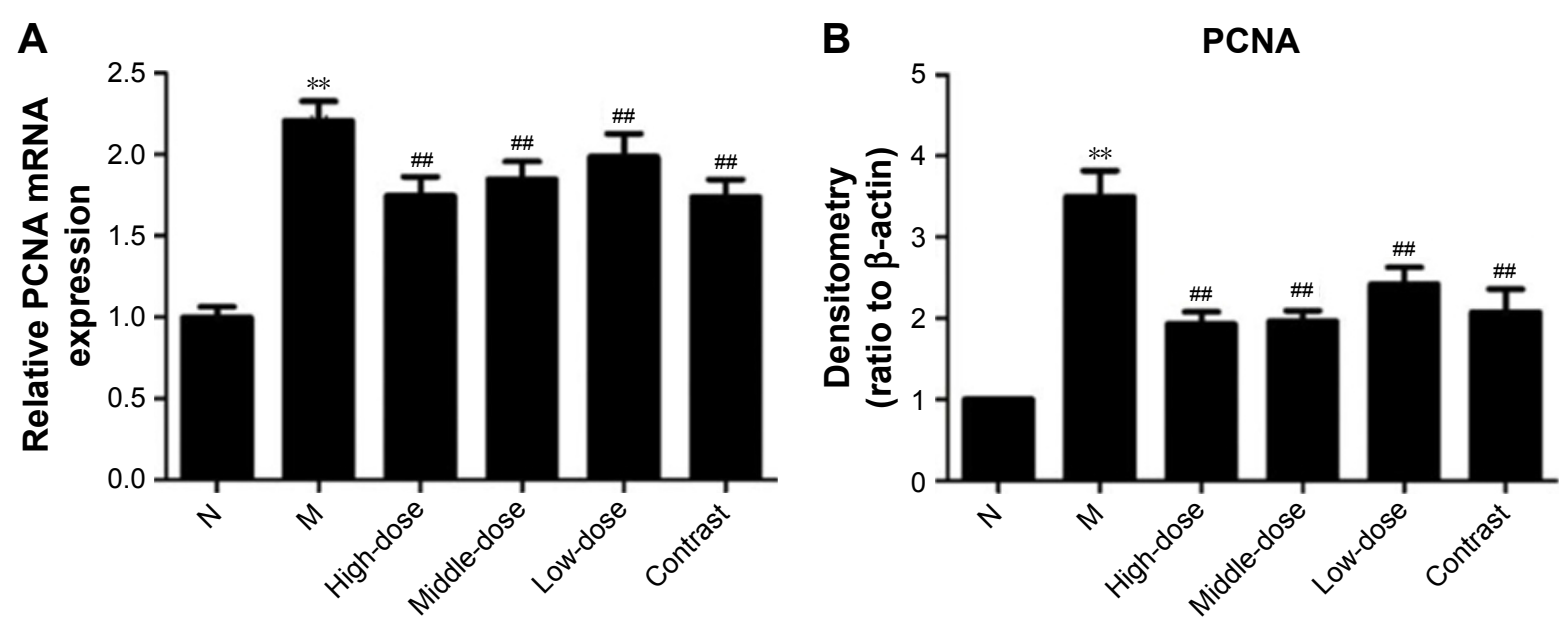

\section{C}

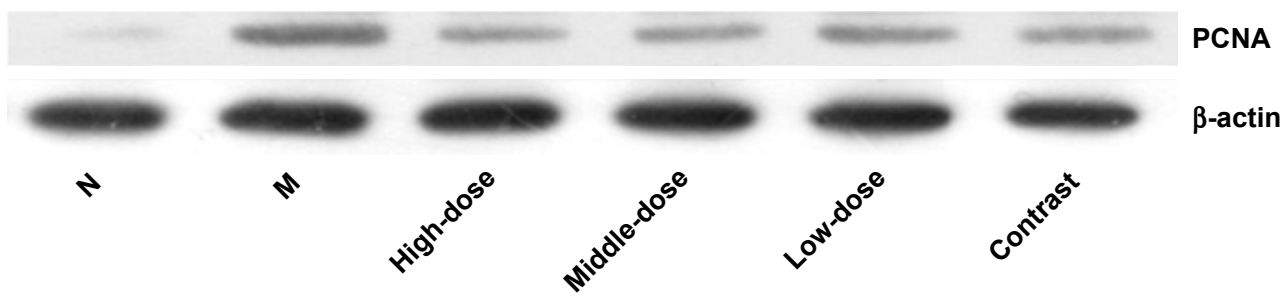

Figure 8 Effect of YPG on the expression of PCNA in BA rats. (A) mRNA levels of PCNA were determined by real-time PCR. (B, C) PCNA protein levels were evaluated by Western blot.

Notes: $\beta$-actin was used as a loading control and for normalization of densitometry data, which are expressed as means \pm SD. $n=3$. $* * P<0.01$ vs healthy control $(N)$; ${ }^{\#} P<0.05$ vs model group (M).

Abbreviations: YPG, Yanghe Pingchuan granules; BA, bronchial asthma.

functions, including proliferation, transcription, apoptosis, cell migration, glucose metabolism, and differentiation, via catalysis of the phosphorylation of the 3-OH position of phosphoinositides and generation of lipids. ${ }^{25,26}$ The PI3K family is divided into four classes; three of which have phosphorylated lipids as their primary targets, while the fourth category (mTOR, ATM, ATR, and DNA-PK) targets phosphorylated proteins. ${ }^{27}$ Growth factors and insulin can activate PI3K by targeting its catalytic membrane subunit close to its substrate (primarily PIP2). PKB contains a C-terminal pleckstrin homology (PH) domain, and binds to membrane-bound PIP3. PI3K can induce the activation of PKB in two steps. First, PIP3 recruits PKB to the cell membrane through binding to the PH domain of PKB, permitting its activation by PDK1. PDK1 contains a PH domain, binds to membrane-bound PIP3, and triggers its activation. Hyperactivity of PKB can promote cell growth, cell proliferation, and metabolism, along with resistance to hypoxia and migration. ${ }^{28}$

PCNA is a necessary cofactor for a critical enzyme in the process of DNA synthesis, and the presence of PCNA significantly increases the rate of DNA synthesis; hence, cell proliferation can be estimated by measurement of PCNA expression. ${ }^{20}$ Airway remodeling, an essential feature of asthma, is due to abnormal proliferation of ASMCs, and an unusual increase in ASMCs may correlate with increased expression of PCNA.

In TCM, asthma is often associated with kidney Yang deficiency. "Chronic lung disease involving the kidney" and the "internal-external relationship between the lung and kidney" are both also essential concepts in TCM. These TCM concepts are based on the consequences of neuroendocrine disturbance and abnormal function of the HPA axis. ${ }^{29}$ Expression levels of EPI and AR can be used as markers of the function of the HPA axis; hence, changes in their levels can be used to evaluate the impact of kidney deficiency on asthma. ${ }^{30}$

In this paper, we aimed to investigate the mechanism of action of YPG in treatment of asthma by evaluation of three relevant aspects: the PI3K signaling pathway, cell proliferation of cells (PCNA), and kidney Yang deficiency. The results of H\&E staining and analysis of IL-6, IL-8, and IL-1 $\beta$ expression indicate that YPG has a therapeutic effect in BA. Moreover, UPLC analysis demonstrated that YPG contains five active ingredients: sinapine thiocyanate, ferulic acid, acteoside, auercetin, schisandrin. Data from Westernblotting, real-time PCR, and ELISAs indicate increased 
expression of PI3K, PKB, PIP2, and PIP3, suggesting that asthma results in enhanced activity of the PI3K pathway. Increased expression of the PI3K pathway can promote cell proliferation and differentiation. Therefore, the PI3K pathway appears to be a leading cause of airway remodeling, and is a potential target for treatment. After treatment with YPG, the levels of PI3K, PKB, PIP2, and PIP3 were significantly decreased, demonstrating that YPG affects airway remodeling through inhibiting the expression of the PI3K signaling pathway. Levels of the cell proliferation marker, PCNA, were elevated in the BA model and reduced after treatment with YPG. These results show that asthma is accompanied by abnormal cell proliferation, accompanied by changes in PCNA expression, indicating that abnormal cell proliferation can cause airway remodeling, and that inhibition of such proliferation by YPG can be used to treat airway remodeling in asthma. Levels of the markers of HPA axis activity and kidney Yang deficiency, EPI and AR, were markedly changed before and after asthma. These results demonstrate reduced function of the HPA axis in asthma, which may contribute to asthma pathogenesis. After treatment with YPG, the expression of EPI and AR was increased, suggesting that this preparation can stimulate the activity of the HPA axis, improve the symptoms of kidney Yang deficiency, and be used for treatment of asthma.

\section{Conclusion}

In conclusion, our study confirms that YPG can be used to treat asthma through blocking the PI3K/PKB pathway, inhibiting the abnormal proliferation of ASMCs, and ameliorating kidney Yang deficiency. This is the first report of a link between kidney Yang deficiency and asthma. The results of this investigation clearly suggest new approaches for asthma treatment by blocking the PI3K/PKB signaling pathway and ameliorating kidney Yang deficiency, and provide strong support for the clinical application of YPG.

\section{Acknowledgments}

LingYu Pan and YanQuan Han are considered co-first authors. This work was supported by grant KJ2015A124 from the Clinical Research Fund of the Anhui University of Chinese Medicine (The key projects of Natural Science Foundation of Anhui University), China. We thank Professor FeiFei Ding for English language editing.

\section{Disclosure}

The authors report no conflicts of interest in this work.

\section{References}

1. Oraka E, Iqbal S, Flanders WD, Brinker K, Garbe P. Racial and ethnic disparities in current asthma and emergency department visits: findings from the National Health Interview Survey, 2001-2010. J Asthma. 2013; 50(5):488-496.

2. Baldwin L, Roche WR. Does remodelling of the airway wall precede asthma. Paediatr Respir Rev. 2002;3(4):315-320.

3. Elias JA, Lee CG, Zheng T, Ma B, Homer RJ, Zhu Z. New insights into the pathogenesis of asthma. J Clin Invest. 2003;111(3):291-297.

4. Sumi Y, Hamid Q. Airway remodeling in asthma. Allergol Int. 2007; 56(4):341-348.

5. Do J, Hwang J, Seo H, Woo W, Nam S. Antiasthmatic activity and selective inhibition of type 2 helper T cell response by aqueous extract of semen armeniacae amarum. Immunopharmacol Immunotoxicol. 2006; 28(2):213-225.

6. Holgate ST. Pathogenesis of asthma. Clin Exp Allergy. 2008;38(6): 872-897.

7. Grigoras A, Grigoras CC, Giusca SE, Caruntu ID, Amalinei C. Remodeling of basement membrane in patients with asthma. Rom J Morphol Embryol. 2016;57(1):115-119.

8. Lazaar AL, Panettieri RA. Airway smooth muscle: a modulator of airway remodeling in asthma. J Allergy Clin Immunol. 2005;116(3): 488-495.

9. Hirst SJ, Martin JG, Bonacci JV, et al. Proliferative aspects of airway smooth muscle. J Allergy Clini Immunol. 2004;114(2 Suppl):S2-S17.

10. Peiris TH, Ramirez D, Barghouth PG, Oviedo NJ. The Akt signaling pathway is required for tissue maintenance and regeneration in planarians. BMC Dev Biol. 2016;16(1):7.

11. Kanao R, Masutani C. Regulation of DNA damage tolerance in mammalian cells by post-translational modifications of PCNA. Mutat Res. 2017;803-805:82-88.

12. Dong J. The relationship between traditional Chinese medicine and modern medicine. Evid Based Complement Alternat Med. 2013;2013: 153148 .

13. Kong L, Zhang H, Cao Y, et al. The anti-inflammatory effects of invigorating kidney and supplementing Qi Chinese herbal formulae in asthma patients. Evid Based Complement Alternat Med. 2017;2017:3754145.

14. Wang Y, Xia LZ, Gui J, Jiang H. Experimental study of protection and its mechanism of Yanghepingchuan granules on asthmatic rats. Pharmacol Clin Chin Mater Med. 2014;30(2):134-137.

15. Li Z, Wang CY, Li XM, Peng B, Hu GJ, Zhang NZ. Effect of Yanghe Pingchuan granules on inflammation and the morphology changes of tissue in guinea pig experimental asthma. Chin Arch Trad Chin Med. 2007;25(11):2246-2249.

16. Liu Y, Zhu HZ. Clinical study on Yang-He-Ping-Chuan granules treated chronic lung function duration particles bronchial asthma airway inflammation. Journal of Chengdu University of TCM. 2014;37(3):52-55.

17. Zhu H, Ji HY, Zhang NZ, Wang S, Chen W, Yang C. Effect of Yanghe Pingchuan granule on airway inflammation of chronic dampness syndrome in bronchial asthma. Clin J Trad Chin Med. 2010;22(4): 310-312.

18. Fuchs O, Bahmer T, Rabe KF, von Mutius E. Asthma transition from childhood into adulthood. Lancet Respir Med. 2017;5:224-234.

19. Bousquet J, Mantzouranis E, Cruz AA, et al. Uniform definition of asthma severity, control, and exacerbations: document presented for the World Health Organization consultation on severe asthma. J Allergy Clin Immunol. 2010;126(5):926-938.

20. Russell RJ, Brightling C. Pathogenesis of asthma: implications for precision medicine. Clin Sci. 2017;131(14):1723-1735.

21. Sumi Y, Hamid Q. Airway remodeling in asthma. Allergol Int. 2007; 56(4):341-348.

22. Kudo M, Ishigatsubo Y, Aoki I. Pathology of asthma. Front Microbiol. 2013;4:263.

23. Prakash YS. Airway smooth muscle in airway reactivity and remodeling: what have we learned? Am J Physiol Lung Cell Mol Physiol. 2013; 305(12):L912-L933. 
24. Burgess JK, Lee JH, Ge Q, et al. Dual ERK and phosphatidylinositol 3-kinase pathways control airway smooth muscle proliferation: differences in asthma. J Cell Physiol. 2008;216(3):673-679.

25. Liu Y, Yang K, Sun X, et al. MiR-138 suppresses airway smooth muscle cell proliferation through the PI3K/AKT signaling pathway by targeting PDK1. Exp Lung Res. 2015;41(7):363-369.

26. Lee Y, Lee J, Kim MH. PI3K/Akt pathway regulates retinoic acid-induced Hox gene expression in F9 cells. Dev Growth Differ. 2014;56(7): $518-525$.

27. Paz-Ares L, Blanco-Aparicio C, Garcia-Carbonero R, Carnero A. Inhibiting PI3K as a therapeutic strategy against cancer. Clin Transl Oncol. 2009;11(9):572-579.
28. Vivanco I, Sawyers CL. The phosphatidylinositol 3-Kinase AKT pathway in human cancer. Nat Revi Cancer. 2002;2(7):489-501.

29. Du YJ, Li B, Zhang HY, et al. Airway inflammation and hypothalamicpituitary-adrenal axis activity in asthmatic adults with depression. J Asthma. 2013;50(3):274-281.

30. Silverman ES, Breault DT, Vallone J, et al. Corticotropin-releasing hormone deficiency increases allergen-induced airway inflammation in a mouse model of asthma. J Allergy Clin Immunol. 2004;114(4): $747-754$.

\section{Publish your work in this journal}

Drug Design, Development and Therapy is an international, peerreviewed open-access journal that spans the spectrum of drug design and development through to clinical applications. Clinical outcomes, patient safety, and programs for the development and effective, safe, and sustained use of medicines are the features of the journal, which has also been accepted for indexing on PubMed Central. The manuscript management system is completely online and includes a very quick and fair peer-review system, which is all easy to use. Visit http://www.dovepress.com/testimonials.php to read real quotes from published authors.

Submit your manuscript here: http://www.dovepress.com/drug-design-development-and-therapy-journal 